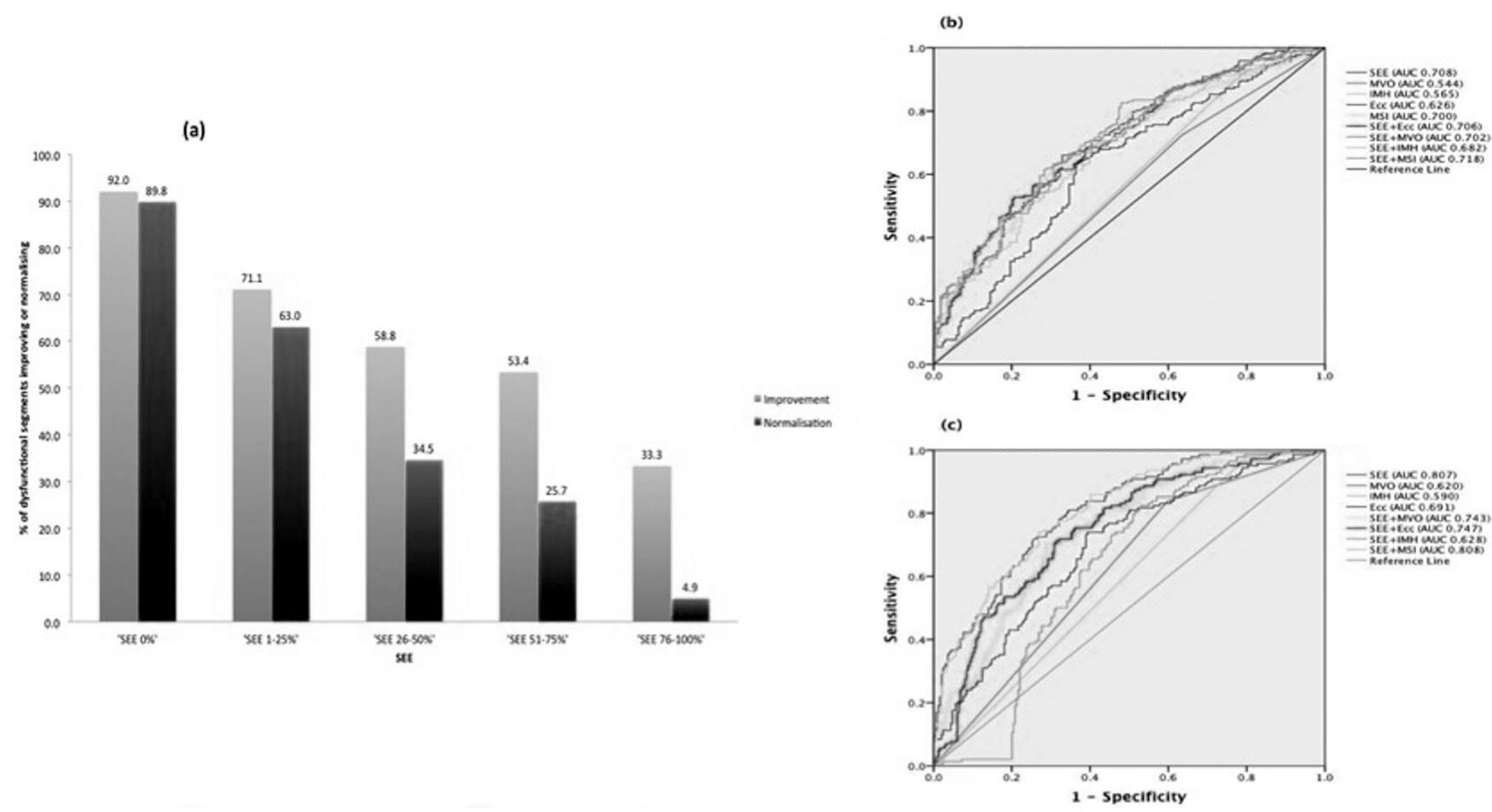

Abstract 18 Figure 1 (a) Recovery in dysfunctional segments at follow-up CMR by SEE. (b) ROC curve of single and combined predictors of segmental improvement in dysfunctional segments. (c) ROC curve of single and combined predictors of segmental normalisation in dysfunctional segments.

IMH, Ecc or MSI did not improve predictive accuracy versus SEE alone (Figure $1 \mathrm{~b}-1 \mathrm{c})$.

Conclusions This is the largest study assessing CMR predictors of segmental recovery in acute STEMI. Baseline SEE was the strongest predictor. Ecc, MSI, MVO and IMH provided no incremental predictive value to SEE. Functional improvement can occur where SEE $>75 \%$.

\section{THE RANDOMISED COMPLETE VS. LESION ONLY PRIMARY PCI TRIAL - CARDIOVASCULAR MRI SUBSTUDY (CVLPRIT-CMR)}

${ }^{1} \mathrm{JN}$ Khan*, ${ }^{2} \mathrm{JP}$ Greenwood, ${ }^{1} \mathrm{SA}$ Nazir, ${ }^{3} \mathrm{M}$ Dalby, ${ }^{4} \mathrm{~N}$ Curzen, ${ }^{5} \mathrm{~S}$ Hetherington, ${ }^{6} \mathrm{DJ}$ Kelly, ${ }^{2} \mathrm{D}$ Blackman, ${ }^{7} \mathrm{~A}$ Ring, ${ }^{4} \mathrm{C}$ Peebles, ${ }^{3} \mathrm{~J}$ Wong, ${ }^{8} \mathrm{M}$ Flather, ${ }^{9} \mathrm{H}$ Swanton, ${ }^{1} \mathrm{AH}$ Gershlick, ${ }^{1}$ GP McCann. 'Department of Cardiovascular Sciences, University of Leicester and the NIHR Leicester Cardiovascular Biomedical Research Unit, University Hospitals of Leicester NHS Trust, Glenfield Hospital, Leicester, LE3 9QP, UK; ${ }^{2}$ Multidisciplinary Cardiovascular Research Centre and the Division of Cardiovascular and Diabetes Research, Leeds Institute of Cardiovascular and Metabolic Medicine (LICAMM), University of Leeds, Leeds, LS2 9JT, UK; ${ }^{3}$ Royal Brompton and Harefield Foundation Trust, Harefield Hospital, Hill End Road, Middlesex UB9 6JH, UK; ${ }^{4}$ University Hospital Southampton NHS Foundation Trust and University of Southampton, Southampton SO16 6YD, UK; ${ }^{5}$ Kettering General Hospital, Rothwell Road, Kettering, NN16 8UZ, UK; ${ }^{6}$ Royal Derby Hospital, Derby DE22 3NE, UK; ' ${ }^{7}$ Leicester Clinical Trials Unit, University of Leicester, Leicester UK and Department of Mathematical Statistics and Actuarial Science, University of the Free State, PO Box 339, Bloemfontein 9300, South Africa; ${ }^{8}$ Norfolk and Norwich University Hospitals NHS Foundation Trust and Norwich Medical School, University of East Anglia Norwich NR4 7TJ, UK; ${ }^{9}$ The Heart Hospital, University College London Hospitals, 16-18 Westmoreland Street, London W1G 8PH, UK

\subsection{6/heartinl-2015-307845.19}

Background Multivessel disease (MVD) occurs in $\sim 40 \%$ of STEMI. Management is controversial. PRAMI and CVLPRIT showed improved clinical outcomes with complete versus infarct-related artery (IRA)-only revascularisation at primary percutaneous coronary intervention (PPCI). However, non-IRA PCI may cause additional infarcts. We aimed to determine whether in-hospital complete revascularisation was associated with increased myocardial injury versus an IRA-only strategy.

Methods Multicentre, prospective, randomised, blinded endpoint trial. STEMI patients with MVD and $<12 \mathrm{hr}$ symptoms were randomised to IRA-only or complete in-hospital PCI. 1.5T CMR was performed acutely (median 3 days post-PPCI) and with adenosine stress at 9 months. The primary CMR endpoint was acute infarct size on late gadolinium imaging. Myocardial salvage index (MSI) was the proportion of non-infarcted area-atrisk. $\mathrm{n}=100$ per group gave $80 \%$ power to detect $\pm 4 \%$ infarct size. The primary clinical outcome was 12 month combined MACE (death, repeat revascularisation, heart failure, MI).

Validation studies optimised infarct, area-at-risk and strain quantification. Full-width half-maximum infarct quantification was more accurate, reproducible and correlated strongest with ejection fraction (LVEF) and infarct characteristics. Otsu's Automated Thresholding most accurately and reproducibly assessed area-at-risk. Compared with tagging, Feature Tracking strain measurement was more robust, quicker, had better interobserver variability and correlated stronger with infarct, area-at-risk and MSI.

Results (summarised in Table 1) 203 patients (98 complete revascularisation, 105 IRA-only) completed acute CMR. The groups were well matched. There was no difference in infarct size, MSI, LVEF, circumferential strain or ischaemic burden between groups. Complete revascularisation patients had increased non-IRA MI at acute CMR (Figure 1). 12 month MACE was reduced in complete revascularisation patients (8.2\% vs. $17.1 \%, \mathrm{p}=0.055$, hazard ratio 0.43$)$.

Conclusions Complete revascularisation in STEMI with MVD leads to a small increase in CMR-detected non-IRA MI, but total infarct size and 12 month MACE are not increased. This 
provides further reassurance that complete revascularisation can be considered at PPCI.

Abstract 19 Table 1 Baseline, angiographic and CMR characteristics

\begin{tabular}{|c|c|c|c|}
\hline Variable & $\begin{array}{l}\text { IRA-only } \\
\text { revascularisation } \\
(n=105)\end{array}$ & $\begin{array}{l}\text { Complete } \\
\text { revascularisation } \\
(\mathrm{n}=98)\end{array}$ & $\mathbf{p}$ \\
\hline \multicolumn{4}{|l|}{ Baseline characteristics } \\
\hline Age (y) & $64.1 \pm 10.8$ & $63.1 \pm 11.3$ & 0.53 \\
\hline Male sex $(n, \%)$ & $83 / 105(79.0)$ & $87 / 98(88.8)$ & 0.06 \\
\hline Anterior infarct $(n, \%)$ & $37 / 105(37.2)$ & $35 / 98(35.7)$ & 0.94 \\
\hline Diabetes Mellitus $(n, \%)$ & $13 / 105(12.4)$ & 15/98 (15.3) & 0.55 \\
\hline \multicolumn{4}{|l|}{ Angiographic markers } \\
\hline TIMI pre $\mathrm{PCI}$ grade $0-2(n, \%)$ & $97 / 105(92.4)$ & $89 / 98(90.8)$ & 0.69 \\
\hline SYNTAX score (total) & $18(14-22)$ & $17.3(13-23.5)$ & 0.81 \\
\hline Symptom-PCI time (TTR, min) & $171(127-268)$ & $192(131-302)$ & 0.20 \\
\hline TIMI post $\mathrm{PCl}$ grade $3(n, \%)$ & 100/105 (95.2) & $89 / 98(90.8)$ & 0.21 \\
\hline \multicolumn{4}{|l|}{ Acute CMR } \\
\hline Time to acute CMR (d) & $2.8(1.8-3.4)$ & $3.0(2.0-4.3)$ & 0.13 \\
\hline LV ejection fraction (\%) & $45.1 \pm 9.5$ & $45.9 \pm 9.9$ & 0.60 \\
\hline Peak LV circumferential strain (Ecc,\%) & $-18.1 \pm 6.0$ & $-18.6 \pm 6.1$ & 0.86 \\
\hline Total infarct size (\% LV mass) & $13.5(6.2-21.9)$ & $12.6(7.2-22.6)$ & 0.57 \\
\hline Patients with $>1$ infarct & $11 / 105(10.5)$ & $22 / 98(22.4)$ & 0.02 \\
\hline Patients $>1$ acute infarct & $5 / 105(4.8)$ & $17 / 98(17.1)$ & 0.004 \\
\hline Myocardial salvage index (\%) & $60.5(40.6-81.9)$ & $58.5(32.8-74.9)$ & 0.14 \\
\hline \multicolumn{4}{|l|}{ Follow-up CMR } \\
\hline Time to follow-up CMR (CMR2, mth) & $9.3(8.9-9.9)$ & $9.4(9.0-10)$ & 0.20 \\
\hline LV ejection fraction (\%) & $50.8 \pm 8.7$ & $49.7 \pm 9.4$ & 0.42 \\
\hline Peak LV circumferential strain (Ecc,\%) & $-23.6 \pm 6.3$ & $-22.5 \pm 6.3$ & 0.28 \\
\hline Total infarct size (\% LV mass) & $7.6(3.2-15.1)$ & $7.3(3.0-14.4)$ & 0.41 \\
\hline Patients with $>1$ infarct (\%) & $9 / 80(11.2)$ & 20/84 (23.8) & 0.035 \\
\hline Presence of ischaemia $(n, \%)$ in all pats & $16 / 77(20.8)$ & $17 / 82(20.7)$ & 0.99 \\
\hline Global ischaemic burden (\%) all pats & $4.3 \pm 11.3$ & $3.4 \pm 8.9$ & 0.81 \\
\hline
\end{tabular}

20 MYOCARDIAL EXTRACELLULAR VOLUME PREDICTS FUNCTIONAL RECOVERY IN ACUTE MYOCARDIAL INFARCTION MORE ACCURATELY THAN THRESHOLDBASED MEASURES OF LATE GADOLINIUM ENHANCEMENT TRANSMURAL EXTENT

A Kidambi*, M Motwani, A Uddin, DP Ripley, AK McDiarmid, PP Swoboda, DA Broadbent, TA Musa, B Erhayiem, JP Greenwood, S Plein. University of Leeds, Leeds, UK

\subsection{6/heartjnl-2015-307845.20}

The transmural extent of late gadolinium enhancement (LGE) CMR predicts functional recovery in acute myocardial infarction (AMI). Automated methods are recommended ${ }^{1}$ to define infarct extent on LGE imaging, such as 'n-standard deviations' (SD) and 'full width at half maximum' (FWHM). These define infarcted myocardium by signal intensity as compared to remote myocardium, which in turn depends on signal-to-noise and contrast. Individual variability in these parameters makes a single thresholding technique unlikely to be universally suitable. Extracellular volume (ECV) estimation by T1-mapping CMR is theoretically less affected by sequence and contrast variations. We compared infarct ECV with threshold-based measures of LGE transmural extent to predict contractile recovery in reperfused AMI.

Consecutive patients with reperfused first ST-elevation AMI underwent acute (day 2) and convalescent (3 months) CMR. Cine imaging, modified Look-Locker inversion T1 mapping natively and 15 min post gadolinium-contrast administration and LGE imaging were performed. Five LGE thresholding techniques were compared: 2, 5 and $6 \mathrm{SD}$, FWHM and a histogram-based technique (Otsu). ${ }^{2}$ The ability of acute infarct ECV to predict improvement in segmental wall motion was compared with these thresholding techniques.

$\mathrm{n}=35(28(80 \%)$ male, age $57 \pm 11$ years). Infarct characteristics are shown in Table 1. ECV showed modest correlation with all threshold measures of LGE $\left(\mathrm{r}^{2}=0.16-0.31, \mathrm{p}<0.01\right)$.
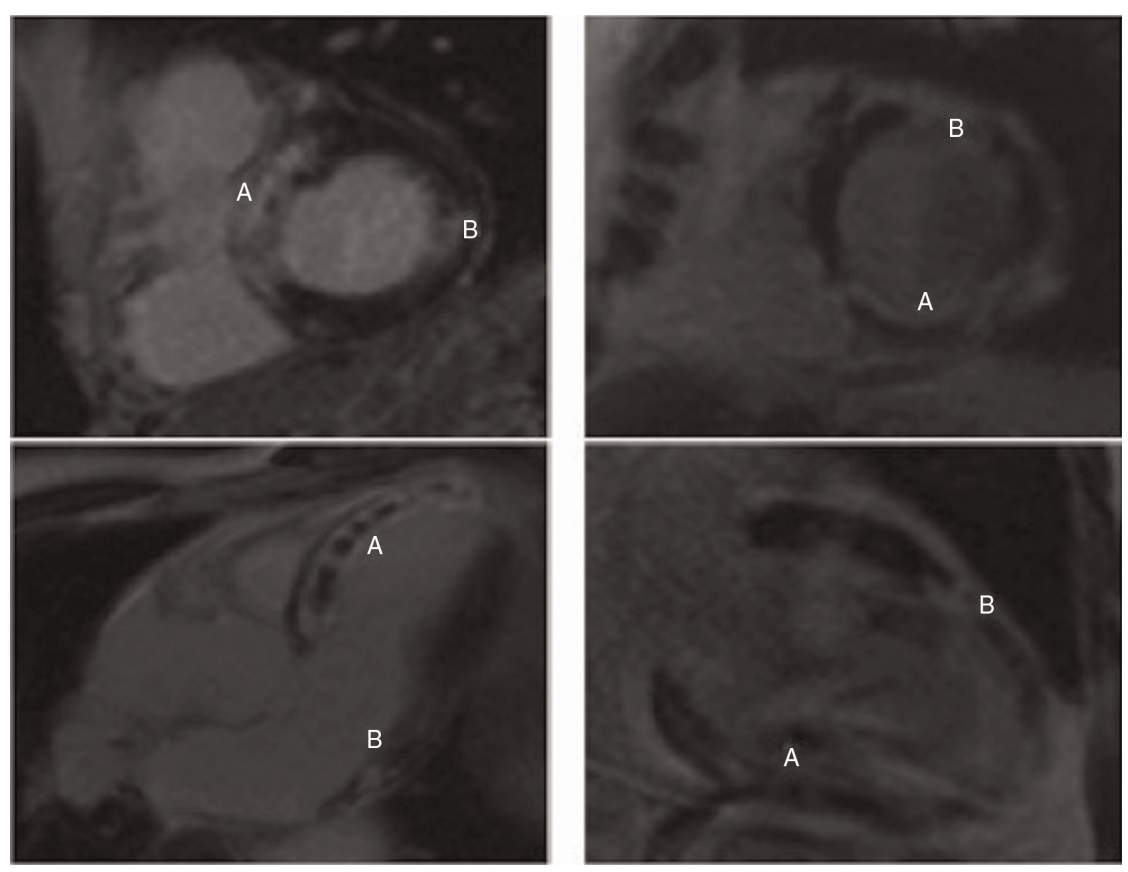

Abstract 19 Figure 1 Multiple infarcts on late gadolinium imaging in complete revascularisation patients The 2 images on left and 2 images on right are 2 different patients.

$A=$ main infarct-related artery territory infarct

$\mathbf{B}=$ infarct in non-infarct related artery territory 